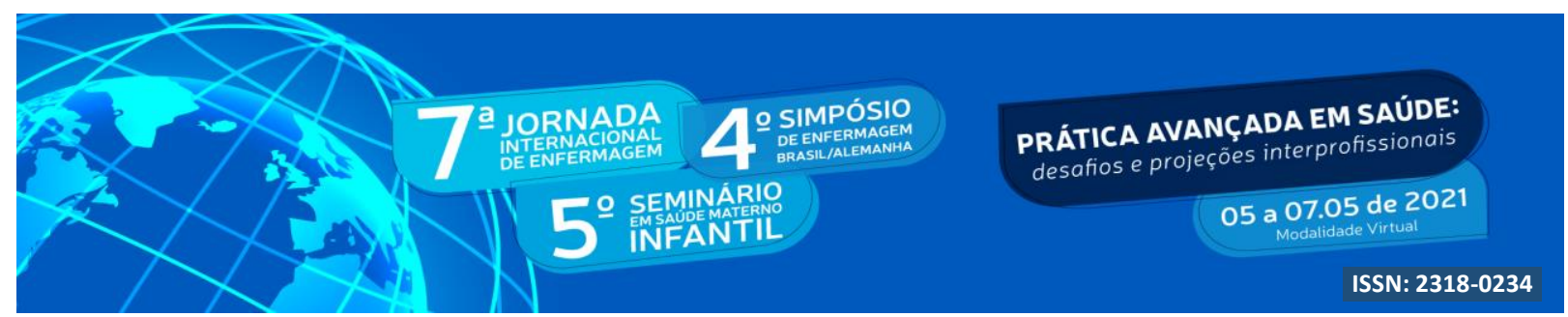

DOI: http://doi.org/10.48195/jie2021-036

\title{
DESAFIOS DO CUIDADO AO PÉ DIABÉTICO NO CENÁRIO DA PANDEMIA DE COVID-19: REFLEXÕES ${ }^{1}$
}

\section{Anna Júlia Pacheco Alves ${ }^{2}$; Isadora Balconi ${ }^{3}$; Adrielli Idalgo Balconi ${ }^{4}$; Luiza Camila Jerke $^{5}$; Vivian de Franceschi Brondani ${ }^{6}$; Maria Denise Schimith ${ }^{7}$}

\begin{abstract}
RESUMO
O presente estudo objetiva refletir acerca do cuidado e dos desafios para com pessoas com pé diabético no cenário da pandemia de COVID-19, as complicações e agravos diante da dificuldade de acesso aos serviços de saúde e a função da enfermagem nesse contexto. Trata-se de um estudo descritivo, teórico-reflexivo, a partir da análise de estudos disponíveis na Biblioteca Virtual de Saúde. Os resultados revelam que estratégias como telemedicina, incentivo ao auto exame dos pés e cuidados regulares, uso de palmilhas de detecção de pressão e temperatura, monitoramento remoto e vídeoconsultas foram eficientes no cuidado às pessoas com pé diabético. Desta forma, a capacitação de profissionais de saúde acerca das mudanças nos cuidados, são fundamentais para enfrentar as barreiras e prestar assistência de qualidade.
\end{abstract}

Palavras-chave: Assistência Integral à Saúde; COVID-19; Diabetes Mellitus; Enfermagem; Pé diabético.

\begin{abstract}
The present study objective to reflect about care and challenges for people with diabetic foot in the context of the COVID-19 pandemic, complications and difficulty of access to health services and the role of nursing in this context. This is a descriptive, theoretical-reflective study, based on the analyses of studies available at the Virtual Health Library. The results reveal that strategies such as telemedicine, encouraging self-examination of the feet and regular care, use of pressure and temperature detection insoles, remote monitoring and video queries were efficient in caring for people with diabetic foot. Therefore, the training of health professionals about changes is essential to confront the barriers and provide quality care.
\end{abstract}

\footnotetext{
${ }^{1}$ Estudo vinculado ao Grupo de Pesquisa Cuidado, Saúde e Enfermagem: Políticas Públicas e Práticas de Cuidado nos diversos cenários de Atenção à Saúde (PraCCeS) da Universidade Federal de Santa Maria (UFSM)/ Iniciação Científica.

${ }^{2}$ Estudante do Curso de Enfermagem. Universidade Federal de Santa Maria. Bolsista do Programa de Educação Tutorial - PET Enfermagem. E-mail: anna.lilo2000@gmail.com

${ }^{3}$ Estudante do Curso de Enfermagem. Universidade Federal de Santa Maria. Bolsista do Programa de Educação Tutorial - PET Enfermagem. E-mail: isadorapbalconi@gmail.com

${ }^{4}$ Estudante do Curso de Enfermagem. Faculdade Integrada de Santa Maria. E-mail: adriidalgobalconi@gmail.com

${ }^{5}$ Estudante do Curso de Enfermagem. Universidade Federal de Santa Maria. E-mail: luizajerke@ gmail.com

${ }^{6}$ Estudante do Programa de Pós-graduação em Enfermagem. Universidade Federal de Santa Maria. E-mail: vivianbrondani@gmail.com

${ }^{7}$ Orientadora. Enfermeira. Professora Adjunta da Universidade Federal de Santa Maria. E-mail:

ma.denise2011@gmail.com 


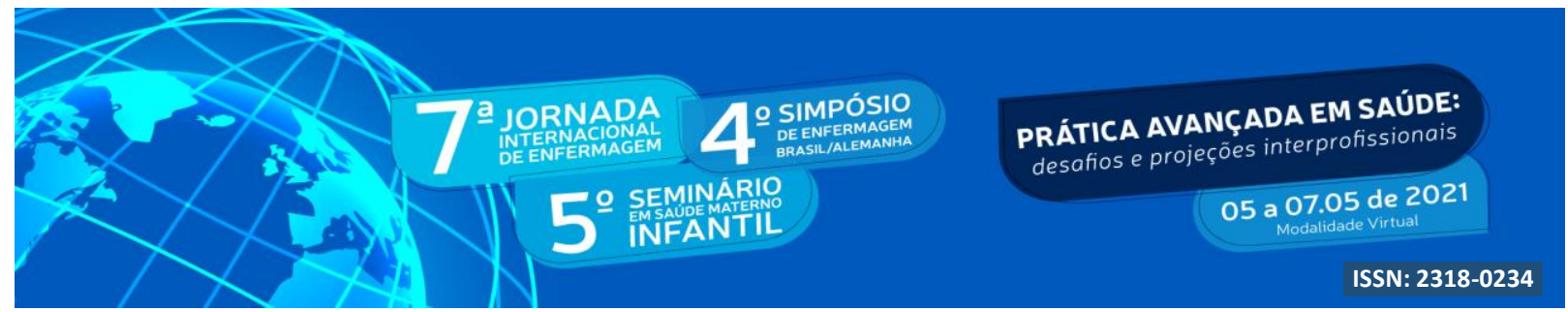

Key Words: COVID-19; Comprehensive Health Care; Diabetes Mellitus; Diabetic Foot; Nursing.

\section{INTRODUÇÃO}

Com os primeiros casos confirmados de "pneumonia viral," no dia 31 de dezembro de 2019, em Wuhan, na República Popular da China, pelo novo coronavírus denominado de Severe Acute Respiratory Syndrome Coronavirus 2 (SARS-CoV-2), causador da doença altamente infecciosa Coronavirus Disease 2019 (COVID-19), confirmada no Brasil, no estado de São Paulo, em 26 de fevereiro de 2020, esta tomou proporções imensuráveis (BRASIL, 2020a). No mundo, os números apontam que, após confirmação do novo coronavírus, houve 107.423.526 casos e 2.360.280 mortes até 12 de fevereiro de 2021. Já no Brasil, até 11 de março de 2021, 11.277 .717 casos acumulados e 272.889 vítimas fatais pela COVID-19 (ORGANIZAÇÃO PAN-AMERICANA DE SAÚDE/ORGANIZAÇÃO MUNDIAL DA SAÚDE, 2021a; BRASIL, 2021).

Com base nesses números alarmantes, em uma pesquisa realizada pela OPAS/OMS (2020), os serviços de saúde passaram por uma reorganização, diminuindo assim o número de profissionais capacitados disponíveis, visto que estes tiveram de ser redirecionados à linha de frente no combate à COVID-19. Dessa forma, a assistência às pessoas com Doenças Crônicas Não Transmissíveis (DCNTs), como câncer, Diabetes Mellitus (DM), Hipertensão Arterial Sistêmica, deixou de ser a grande prioridade nos centros de saúde, tornando-se os usuários com DCNT mais suscetíveis aos agravos advindos pela COVID-19, em caso de infecção, pelo acompanhamento inadequado de suas comorbidades.

Segundo estimativas da Sociedade Brasileira de Diabetes (2020), 16 milhões de brasileiros convivem com a doença. E, cerca de $20 \%$ da população com diabetes desenvolvem algum problema nos pés no decorrer da vida. Ainda, considera que "a diabetes é a primeira causa de amputações não traumáticas em membros inferiores no mundo. É estimada uma amputação a cada 20 segundos".

Portanto, relativo à DM, causa mais comum de cegueira, diálise e amputações, o pé diabético encontra-se entre as complicações mais frequentes, especialmente em pessoas com a doença não controlada. Trata-se de uma complicação crônica nos pés resultando em condições 


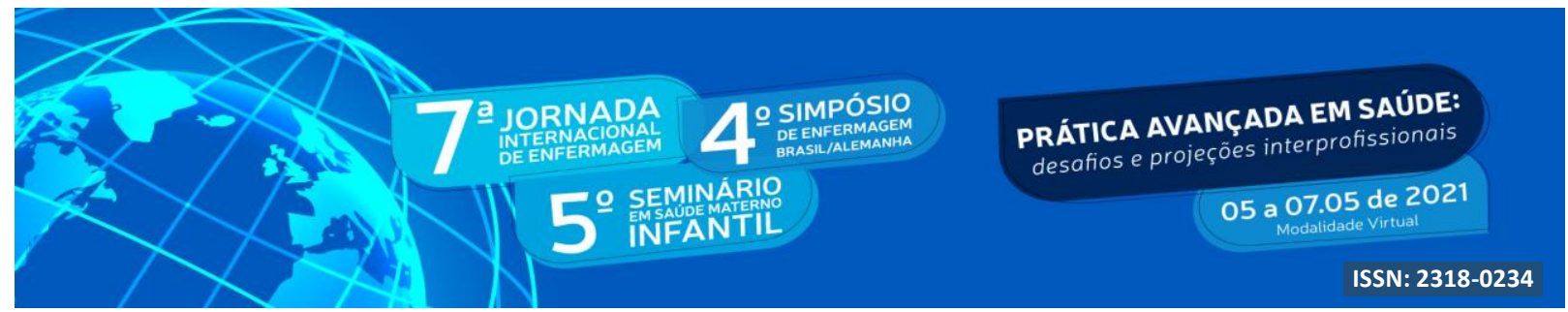

diversas para as pessoas acometidas, desde problemas na circulação e sensibilidade, feridas crônicas, infecções e até amputações de membros inferiores (BRASIL, 2016a; SOCIEDADE BRASILEIRA DE DIABETES, 2017).

Nesse ínterim, a neuropatia periférica e problemas na circulação sanguínea são os principais riscos para o desenvolvimento da doença, devido à redução da sensibilidade protetora e da oclusão das artérias dos membros inferiores pela aterosclerose, podendo haver ferimentos sem perceber, dificuldade de cicatrização e ausência dos pulsos tibial posterior e pedioso dorsal. Ainda, as modificações musculares e articulares podem ocasionar deformidade nos pés, elevando o risco de ulcerações (BRASIL, 2016a).

Nesse contexto, é possível observar a imprescindibilidade em reconhecer as condutas dos profissionais diante do cuidado ao paciente com pé diabético. A enfermagem desempenha esse papel primordial, tanto na prevenção, quanto no tratamento dessa patologia (BRASIL, 2013). Por isso, frente aos desafios decorrentes da pandemia, é importante conhecer as atuais condutas profissionais para evitar lesões e amputações no que se refere às pessoas com diabetes com risco para o pé diabético.

Dessa forma, sabe-se que a assistência ao pé diabético deve abranger o modelo de atenção integral, a fim de prevenir e restaurar a funcionalidade da extremidade afetada (BRASIL, 2016b). Logo, no cenário de pandemia, devido à reorganização dos serviços de saúde e o fluxo de atendimento, mostra-se significativa a abordagem da temática para o conhecimento acerca das complicações, sobretudo os riscos, o cuidado e os desafios advindos dessas mudanças.

\section{OBJETIVO}

Refletir acerca do cuidado e dos desafios para com pessoal com pé diabético no cenário da pandemia de COVID-19, as complicações e agravos diante da dificuldade de acesso aos serviços de saúde e a função da enfermagem nesse contexto.

\section{METODOLOGIA}




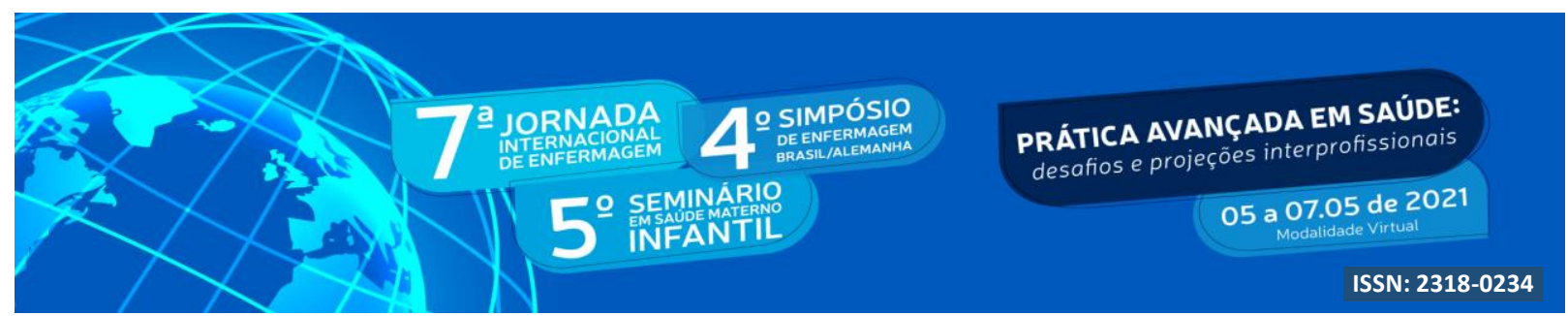

Trata-se de um estudo descritivo, do tipo teórico-reflexivo, a partir da análise de estudos selecionados nas bases de dados Medical Literature Analysis and Retrieval System Online (MEDLINE), Base de Dados de Enfermagem (BDENF) e da Literatura Latino Americana e do Caribe de Informações em Ciências da Saúde (LILACS), via Portal Regional da Biblioteca Virtual em Saúde (BVS).

O método teórico-reflexivo mostra-se próximo à abordagem qualitativa, uma vez que permite a análise de dados e materiais teóricos a partir da busca bibliográfica. Além disso, na formação acadêmica, instiga a construção de novas formas de compreender e agir, fomentando o olhar de transformação das realidades existentes (LIMA, et. al., 2014; NETTO; SILVA; RUA, 2018). Nesse sentido, apresenta-se como uma estratégia importante no desenvolvimento de competências para a promoção da saúde e prevenção de doenças.

A busca foi realizada no mês de março de 2021, por estudantes da graduação e pósgraduação em enfermagem. Os descritores utilizados nas estratégias de busca foram extraídos nos Descritores em Ciências da Saúde (DeCS) e, após testagem das estratégias nas bases de dados, resultou na seguinte estratégia: "pé diabético" AND "COVID-19”. Utilizaram-se como critério de inclusão: estudos disponibilizados na íntegra on-line, gratuitamente, nos idiomas inglês, português ou espanhol, com recorte temporal de um ano, em decorrência dos primeiros casos e da declaração de pandemia pela Organização Mundial da Saúde, que respondessem ao objetivo da pesquisa.

A estratégia resultou em 22 estudos, sendo 20 oriundos da base MEDLINE, 1 da BDENF e 1 da LILACS. Logo, os artigos duplicados foram contabilizados apenas uma vez e após a análise, conforme os critérios de inclusão, chegou-se a um resultado de 16 estudos, que foram apreciados mediante leitura na íntegra.

\section{RESULTADOS E DISCUSSÃO}

A partir da análise dos estudos incluídos, evidenciou-se que o distanciamento social foi considerado a medida mais efetiva para evitar a exposição ao vírus. Todavia, no que remete às pessoas com DM, produziu mudanças nos hábitos de vida, como inatividade física, maior consumo de alimentos processados, redução do acesso à assistência e ao controle 


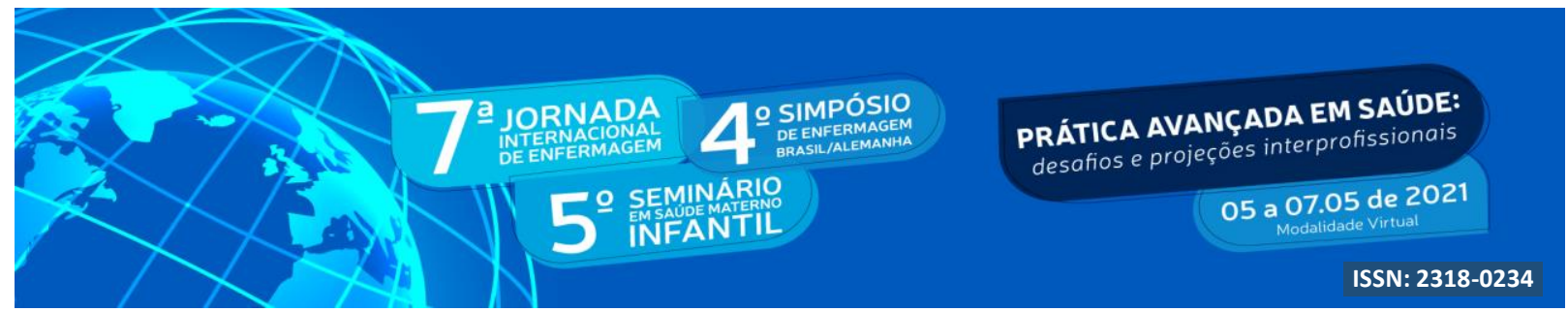

glicêmico. O estudo de Cerqueira et al. (2020) aborda que estratégias visando enfrentar essas peculiaridades têm sido discutidas, bem como o monitoramento de forma remota, triagem por telemedicina com interlocução entre profissional e pessoa com a lesão.

Na infecção por COVID-19, os indivíduos com neuropatia diabética ou doença arterial periférica possuem uma resposta inflamatória adicional, que se torna mais exuberante no caso de úlceras em atividade, implicando no aumento da glicemia, resultando em desordem no sistema imunológico, tornando-os mais suscetíveis à infecção (PAPANAS; PAPACHRISTOU, 2020; CERQUEIRA et al., 2020; LIU et al., 2020a). Estudos revelam que os testes de Proteína C-reativa (PCR) e Procalcitonina (PCT) de pacientes com COVID-19 e DM, apresentaram maiores índices do que os de pacientes sem DM, visto que o sistema imunológico mostrou-se fortemente inibido (CHEN et al., 2020). Além disso, o risco aumentado à COVID-19 em pessoas com Diabetes, ocorre porque grande parte possuem outras comorbidades, levando-os a altas taxas de internação, paralelo ao aumento do tempo de cicatrização de úlceras por pé diabético, acréscimo da taxa de amputação e de mortalidade (CHEN et al., 2020; NAJAFI, 2020; OROPALLO et al., 2021).

Desde o surgimento do Coronavírus, o intuito dos serviços de saúde é reservar leitos hospitalares às pessoas contaminadas, o que torna um impasse para o cuidado ao pé diabético. Isso porque se trata de uma das complicações da DM que mais envolve cuidados face a face, como orientações, desbridamento, curativos e outras modalidades de atendimentos. Logo, o cuidado e a prevenção precisam ser aprimorados, tendo em vista que o acompanhamento profissional não ocorre da mesma forma (NAJAFI, 2020; JALY et al., 2020; SHIN et al., 2020).

Estudos indicam que profissionais que realizavam assistência direta ao tratamento do pé diabético foram realocados para outras áreas, como enfermeiros. Logo, a redução desses profissionais promoveu uma lacuna na prestação de serviços, no agravamento de lesões e podem aumentar as amputações (JALY et al., 2020). Pesquisa realizada na Inglaterra revelou que a incidência de úlceras em pés diabéticos têm apresentado variação mês a mês, com média, nos últimos 2 anos, de 48 novas úlceras/mês. No entanto, em abril de 2020 quando comparado ao ano de 2019, ocorreu a redução de 52\% desses casos, isso porque há um menor número de pessoas procurando os serviços (LIPSCOMB et al., 2020). As abordagens de 


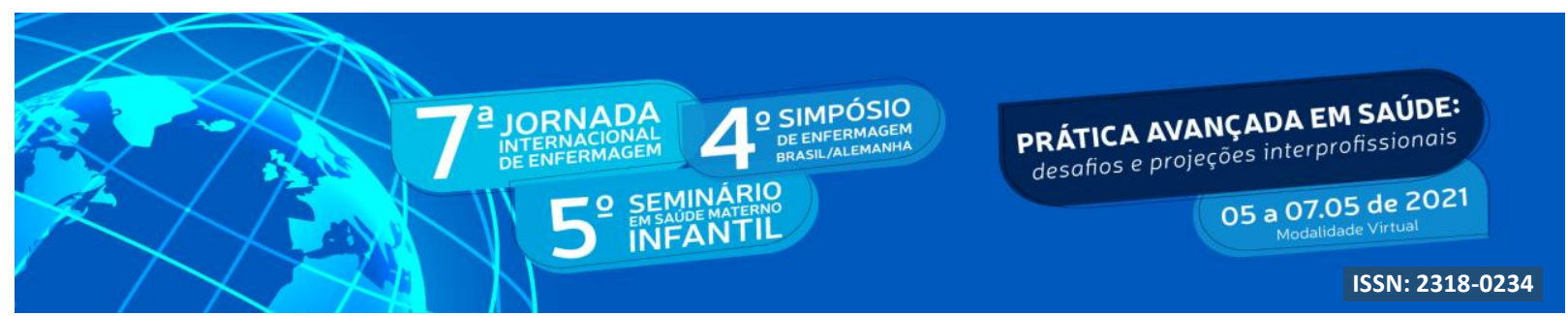

Mader (2020) vão ao encontro dessa premissa, de que o número de pacientes para consultas ambulatoriais reduziu, devido a muitos cancelamentos pelo risco de contaminação pelo Coronavírus.

Além dos desafios da evolução do pé diabético no contexto da pandemia, outros fatores ocorreram paralelamente, como o transporte aos serviços, a logística devido à reorganização dos profissionais, a nova rotina dos pacientes para prevenir o contágio. Ainda, conforme (TAO et al., 2020), estudo realizado na China, o tratamento cirúrgico de úlceras também passou por obstáculos. Desse modo, a fim de tratar as necessidades das pessoas com DM e garantir a segurança de todos em relação à COVID-19, medidas foram adotadas, como a capacitação dos profissionais para evitar a contaminação, realização de exames diários, sobretudo tomografias, triagem para isolar pessoas com estado febril ou não e com infecção ou não, separação por alas de acordo com o quadro clínico.

A fim de enfrentar os impactos da pandemia, os estudos citam diferentes estratégias, sobretudo a educação em saúde e utilização de recursos on-line, consultas de telemedicina, incentivo ao auto exame dos pés e cuidados regulares, uso de palmilhas de detecção de pressão e temperatura, monitoramento remoto, vídeo-consultas (SHIN et al., 2020; KYROU; ROBBINS; RANDEVA, 2020; MADER, 2020; BOULTON, 2021).

Para manter a qualidade do serviço prestado e a atenção integral à saúde, evitar visitas, deslocamentos e exposição desnecessária desses pacientes ao vírus, adotou-se protocolos de triagem e serviços de telessaúde/telemedicina em diversos países. (BOULTON, 2021; RATLIFF et al., 2020; OROPALLO et al., 2021). Conforme, Ratliff et al. (2020), os serviços de atendimento virtual consideram cuidados urgentes de baixo risco, pacientes negativos para COVID-19, facilitando encaminhamentos apropriados, assim como o suporte e gerenciamento de condições crônicas, acompanhamento de pacientes de instituições de longa permanência, pós-operatório e hospitalização, para minimizar reinternações, assim como a educação e treinamento para profissionais de saúde.

Com triagens e estratificação de risco, os países que adotaram a telemedicina consideraram que o paciente grave deveria receber atendimento hospitalar; em casos moderados, em clínicas ambulatoriais ou consultórios; e em casos de baixo risco, atendimentos domiciliares ou por telemedicina após uma primeira avaliação (BOULTON, 


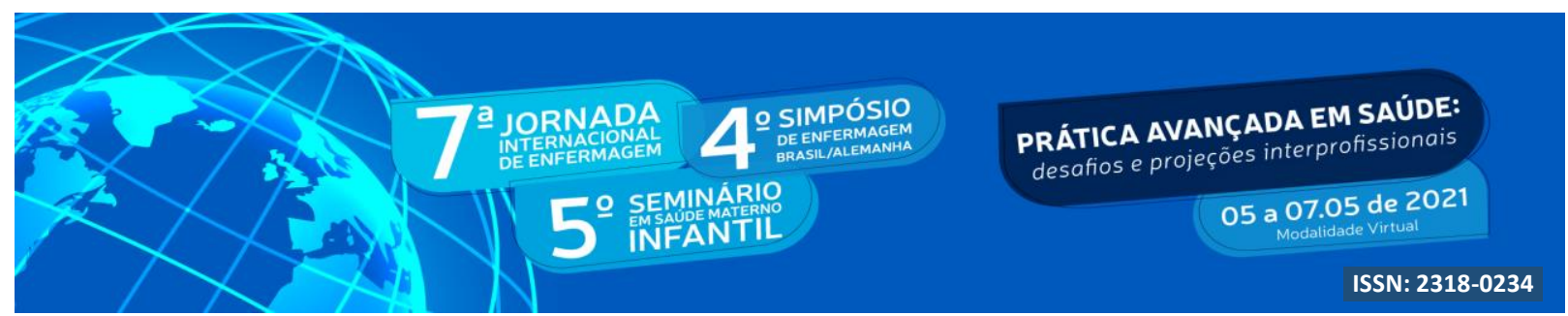

2021; RATLIFF et al., 2020; OROPALLO et al., 2021; LIU et al., 2020b; MELONI et al., 2020). Por meio das avaliações virtuais, realizou-se a educação em saúde dos pacientes, com o objetivo de ofertar maior autonomia no cuidado, como monitoramento de glicose, apoio à saúde mental, auxílio na troca de curativos e limpeza da ferida. Todo esse cuidado domiciliar pode ser monitorado por meio de fotos e vídeos das feridas em diversos ângulos, a cada troca de curativos para acompanhamento do processo de cicatrização ou possíveis agravos (LIU et al., 2020b; MELONI et al., 2020).

Embora a telessaúde minimize a exposição dessa população de risco à COVID-19, deve considerar que nem todos os pacientes possuem experiência e domínio das tecnologias de comunicação, necessitando do amparo de familiares ou de agentes comunitários em casos de idosos sem amparo parental (LIU et al., 2020b; MELONI et al., 2020). Dessa forma, para que essa tecnologia seja benéfica e atinja seus objetivos, deve-se considerar todo contexto social, econômico, domínio tecnológico, acesso à internet e a elegibilidade do paciente que será por esta assistido.

De acordo com Kyrou; Robbins; Randeva (2020) face às particularidades que permeiam tanto à COVID-19 quanto ao Pé diabético, os profissionais precisam manter-se atualizados sobre essas questões, diretrizes, estudos, planos de manejo conforme necessário, visando a melhor assistência possível no cenário existente. Ainda, pesquisadores citam que a telemedicina pode permanecer após pandemia, como um auxílio extra para os profissionais, tendo em vista que muitos pacientes gostam da flexibilidade da tecnologia, mesmo que não substitua a assistência direta (NAJAFI, 2020; MADER, 2020).

\section{CONCLUSÃO}

Em suma, a maior parte dos estudos analisados referem-se a não negligência do pé diabético e suas potenciais complicações durante a pandemia, assim como a relevância do monitoramento contínuo e o exame minucioso dos pés de pessoas com DM, a fim de evitar possíveis lesões ou agravos. Por isso, torna-se evidente a importância do conhecimento técnico-científico, inovação e a atualização acerca do assunto por parte dos profissionais da saúde, não somente da Enfermagem, mas de toda a equipe, a fim de prestar assistência de 


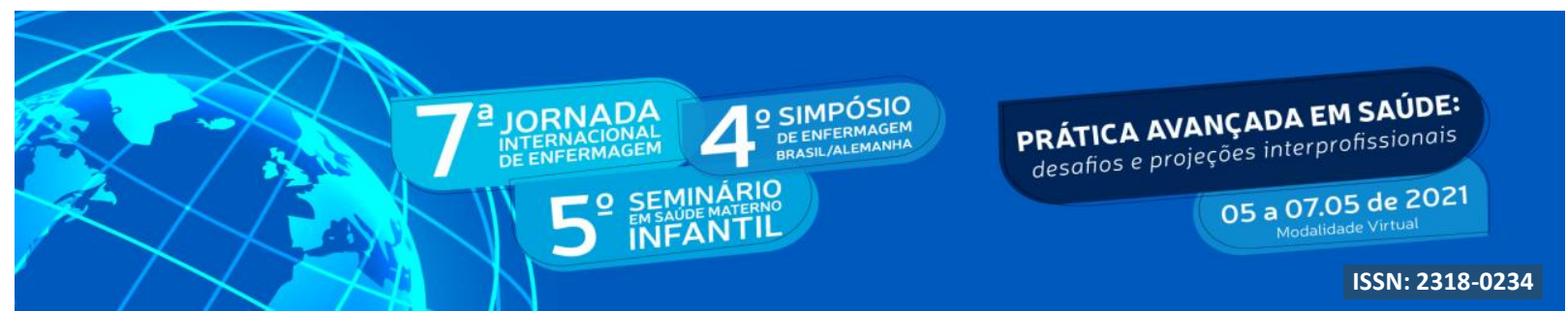

qualidade e de forma integral, conforme as possibilidades do cenário decorrente da pandemia.

Dessa forma, esse novo contexto contribuiu para que novas tecnologias fossem implantadas no tratamento da doença, como a telessaúde, a triagem de risco e as palmilhas de detecção de pressão e temperatura. Estas, mostram-se importantes à assistência ao pé diabético, auxiliando os serviços de saúde para garantir a integralidade do cuidado.

\section{REFERÊNCIAS}

BOULTON, A. J. M. Diabetic Foot Disease during the COVID-19 Pandemic. Medicina (Kaunas),. Reino Unido, 2021, v. 57, n. 2, p. 2-9. Disponível em: https://www.mdpi.com/1648-9144/57/2/97/htm.

BRASIL. Ministério da Saúde. Biblioteca Virtual em Saúde. Pé diabético. 19 de fevereiro de 2016. Página inicial: dicas em saúde. Brasília: Ministério da Saúde, 2016b. Disponível em: https://bvsms.saude.gov.br/dicas-em-saude/2210-pe-diabetico.

Ministério da Saúde. Secretaria de Atenção à Saúde. Departamento de Atenção Básica. Manual do pé diabético: estratégias para o cuidado da pessoa com doença crônica. Brasília, DF: Ministério da Saúde, 2016a, 62p.

Ministério da Saúde. Secretaria de Atenção à Saúde. Departamento de Atenção Básica. Estratégias para o cuidado da pessoa com Doença Crônica: Diabetes Mellitus. (Cadernos de Atenção Básica n. 36). Brasília, DF: Ministério da Saúde, 2013, 160p.

Ministério da Saúde. Secretaria Estadual de Saúde. COVID-19 no Brasil. Dados até 11/03/2021. Página inicial coronavírus COVID-19: Ministério da saúde, 2021a. Disponível em: https://susanalitico.saude.gov.br/extensions/covid-19_html/covid19_html.html.

Ministério da Saúde. Resposta Nacional e internacional de enfrentamento ao novo coronavírus. Página inicial - gov.br. Brasília: Ministério da Saúde, 2020a. Disponível em: https://coronavirus.saude.gov.br/linha-do-tempo/.

CERQUEIRA, M. M. B. F. et al. Propostas de cuidados ao indivíduo com pé diabético em tempo de pandemia do COVID-19 no Brasil. Acta Paul. Enferm. São Paulo, 2020, n. 33, v. 34, p. 1-5. Disponível em: https://acta-ape.org/article/propostas-de-cuidados-ao-individuocom-pe-diabetico-em-tempo-de-pandemia-do-covid-19-no-brasil/.

CHEN, D. et al. The adverse effects of novel coronavirus on diabetic foot patients A protocol for systematic review and meta analysis. Medicine (Baltimore). China, 2020, v. 99, n. 43, p. 1-4. Disponível em: https://journals.lww.com/md- 


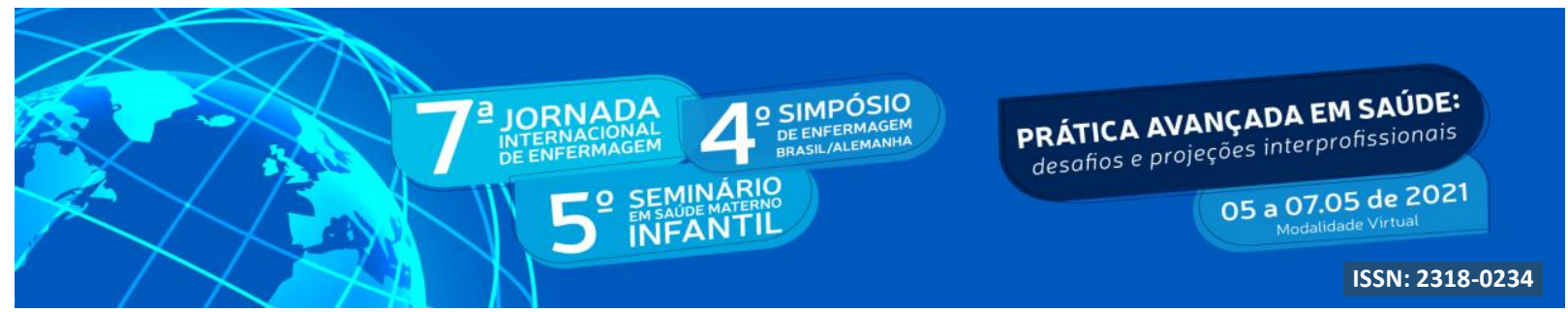

journal/Fulltext/2020/10230/The_adverse_effects_of_novel_coronavirus_on.57. aspx.

JALY, I. et al. Redefining diabetic foot disease management service during COVID-19 pandemic. Diabetes Metab Syndr. United Kingdom, 2020, v. 14, n. 5, p. 833-838. Disponível em: https://www.ncbi.nlm.nih.gov/pmc/articles/PMC7289094/.

KYROU, L.; ROBBINS, T.; RANDEVA, H. S. COVID-19 and diabetes: No time to drag our feet during an untimely pandemic. Journal of Diabetes and Its Complications. 2020, v. 34, n. 9: 107621. Disponível em: https://www.ncbi.nlm.nih.gov/pmc/articles/PMC7228704/.

LIMA, A. C. S. et al. O desafio do conhecimento. Revista Eletrônica Inter- Legere. (ISSN 1982 - 1662). Rio Grande do Norte, 2014, n. 14, p. 1-8. Disponível em: https://periodicos.ufrn.br/interlegere/article/download/4873/4272/.

LIPSCOMB, D. et al. Diabetic foot ulceration in COVID-19 lockdown: cause for concern or unexpected benefit?. Diabetic Medicine. United Kingdom, 2020, v. 37, p. 1409-1410. Disponível em: https://onlinelibrary.wiley.com/doi/full/10.1111/dme.14340.

LIU, C. et al. An internet-based algorithm for diabetic foot infection during the COVID-19 pandemic. Journal of Foot and Ankle Research. China, 2020b, v. 13, n. 37, p. 2-4. Disponível em: https://jfootankleres.biomedcentral.com/articles/10.1186/s13047-020-00405$\mathrm{z}$.

LIU, C. et al. The COVID-19 Outbreak Negatively Affects the Delivery of Care for Patients With Diabetic Foot Ulcers. Diabetes Care. China, 2020a, v. 43, n. 10, p. 125-126. Disponível em: https://care.diabetesjournals.org/content/diacare/43/10/e125.full.pdf.

MADER, J. K. Personal Experiences With Coronavirus Disease 2019 and Diabetes: The Time for Telemedicine is Now. Journal of Diabetes Science and Technology. 2020, v. 14, n. 4, p. 752-753. Disponível em: https://journals.sagepub.com/doi/full/10.1177/1932296820930289.

MELONI, M. et al. Management of diabetic persons with foot ulceration during COVID-19 health care emergency: Effectiveness of a new triage pathway. Diabetes Research and Clinical Practice. Europa, 2020, v. 165. Disponível em: https://www.diabetesresearchclinicalpractice.com/article/S0168-8227(20)30495-2/fulltext.

NAJAFI, B. Post the Pandemic: How will COVID-19 Transform Diabetic Foot Disease Management?. Journal of Diabetes Science and Technology. Houston, 2020, v. 14, n. 4, p. 764-766. Disponível em: https://journals.sagepub.com/doi/pdf/10.1177/1932296820930290.

NETTO, L.; SILVA, K. L.; RUA, M. S. Prática reflexiva e formação profissional: aproximações teóricas no campo da saúde e da enfermagem. Escola Anna Nery. Rio de Janeiro, 2018, v. 22, n. 1, p. 1-6. Disponível em: https://www.scielo.br/scielo.php?script=sci arttext\&pid=S1414-

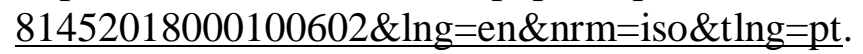




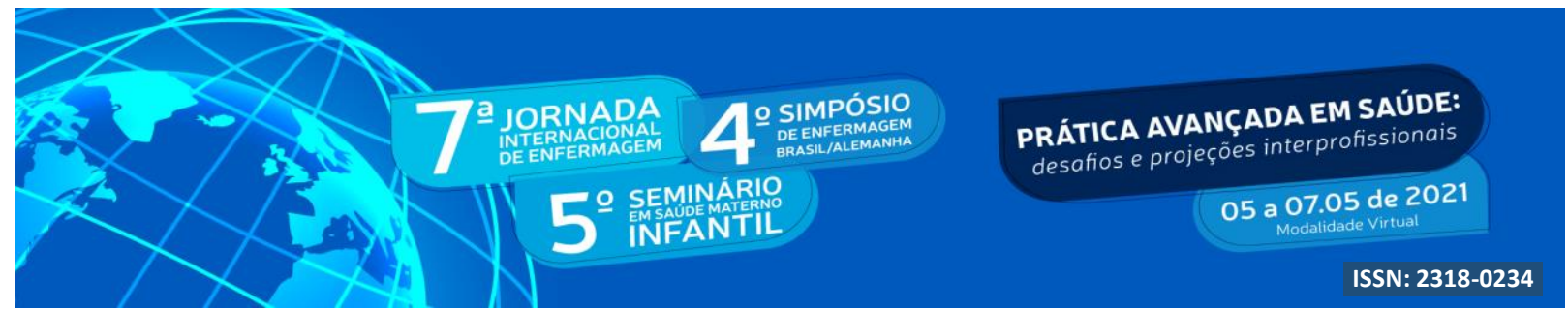

ORGANIZAÇÃO PAN-AMERICANA DA SAÚDE. Organização Mundial da Saúde. Página inicial: determinantes sociais e riscos para a saúde, Doenças Crônicas Não Transmissíveis e Saúde Mental. COVID-19 afeta funcionamento de serviços de saúde para doenças crônicas não transmissíveis nas Américas. 17 de junho de 2020.

ORGANIZAÇÃO PAN-AMERICANA DA SAÚDE. Organização Mundial da Saúde. Folha informativa COVID-19 - Escritório da OPAS e da OMS no Brasil. 12 de fevereiro de 2021. Página inicial: OPAS/OMS, 2021. Disponível em: https://www.paho.org/pt/covid19.

OROPALLO, A. et al. Wound care during the COVID-19 pandemic: improving outcomes through the integration of telemedicine. Journal of Wound Care, Estados Unidos da América (EUA). 2021, v. 30, n. 2, p. 12-17. Disponível em: https://www.magonlinelibrary.com/doi/full/10.12968/jowc.2021.30.Sup2.S12.

PAPANAS, N.; PAPACHRISTOU, S. COVID-19 and Diabetic Foot: Will the Lamp Burn Bright?. The International Journal of Lower Extremity Wounds. Greece, 2020, v. 19, n. 2, p. 111. Disponível em: https://journals.sagepub.com/doi/full/10.1177/1534734620921382.

RATLIFF, C. R. et al. Telehealth for Wound Management During the COVID-19 Pandemic: Case Studies. J Wound Ostomy Continence Nurs. 2020, [S. 1.], v. 47, n. 5, p. 445-449. Disponível em: https://www.ncbi.nlm.nih.gov/pmc/articles/PMC7722288/.

SHIN, L. et al. Saving the Diabetics Foot During the COVID-19 Pandemic: A Tale of Two Cities. Diabetes Care. 2020, v. 43, n. 8, p. 1704-1709. Disponível em:

https://care.diabetesjournals.org/content/43/8/1704.

Sociedade Brasileira de Diabetes (SBD). A enfermagem no cuidado dos pacientes com diabetes será tema do Dia Mundial do Diabetes. 12 de maio de 2020. Disponível em: https://www.diabetes.org.br/publico/noticias/2082-a-enfermagem-no-cuidado-dos-pacientescom-diabetes-sera-tema-do-dia-mundial-do-diabetes-2020.

Sociedade Brasileira de Diabetes (SBD). Diretrizes da Sociedade Brasileira de Diabetes 2017-2018. São Paulo: Editora Clannad, 2017, 383 p.

Sociedade Brasileira de Diabetes (SBD). Página inicial: covid-19/ diabetes. Sociedade Brasileira de Diabetes lança manual para cuidado com o pé diabético durante a pandemia. 30 de julho de 2020. Disponível em: https://www.diabetes.org.br/sociedadebrasileira-de-diabetes-lanca-manual-para-cuidados-com-o-pe-diabetico-durante-a-pandemia/.

TAO, F. et al. Surgical treatment of diabetic foot ulcers during the COVID-19 pandemic in China. Journal of Diabetes and Its Complications. Ásia, 2020, v. 34, n. 9, p. 1-6. Disponível em: https://www.ncbi.nlm.nih.gov/pmc/articles/PMC7224648/. 